\title{
La importancia de la asociatividad para el desarrollo
}

\section{The importance of associativity for development}

María Auxiliadora Guerrero Bejarano, MBA

Universidad Internacional del Ecuador, Ecuador

Ing. Josué André Villamar Cobeña

Universidad Internacional del Ecuador, Ecuador

Autor para correspondencia: jovillamarco@internacinal.edu.ec,maguerrerobe@uide.edu.ec

Fecha de recepción: 10 de Septiembre de 2016 - Fecha de aceptación: 01 de Noviembre de 2016

\section{Resumen}

El siguiente trabajo de investigación, presenta una perspectiva integral de los factores que intervienen en los procesos de asociatividad comunitaria, y su relación e importancia con el entorno en el que es aplicado, con la finalidad de proponer una estrategia que permita mejorar la asociatividad en el marco del desarrollo comunitario. A partir de los resultados se logró concluir la importancia de la relación complementaria que existe entre los factores económicos y no económicos que intervienen en los procesos de asociatividad comunitaria y además se logró obtener una estrategia integral que estimule ambos factores en pro del desarrollo económico y social de nuestro objeto de estudio, la comuna de San Rafael en la Provincia de Santa Elena, Ecuador.

Palabras claves: asociatividad; comunitaria; factores; desarrollo económico; estrategias

\begin{abstract}
The following research paper presents a comprehensive overview of the factors involved in the community associations processes, and its relationship and importance to the environment in which it is applied, in order to propose a strategy to enhance partnership in the under the community development. This research was able to conclude the importance of the complementary relationship between economic and non-economic factors involved in the processes of community association and also managed to secure a comprehensive strategy to stimulate both factors for economic and social development our object of study, the municipality of San Rafael in the Province of Santa Elena, Ecuador.
\end{abstract}

Key words: associativity; communitarian; factors; economic development; strategies 


\section{Introducción}

El Ecuador es considerado uno de los 10 países más biodiversos del mundo, no solo por su variedad de flora y fauna, si no también, por la gran cantidad de diversidad cultural presente en todo su territorio (Biocomercio del Ecuador, 2015). Dentro de esta diversidad cultural, se destacan las culturas ancestrales indígenas de la sierra, de la amazonia ecuatoriana y de la costa. La mayor cantidad de asentamientos descubiertos de culturas autóctonas en la región costera se encuentran en la provincia de Santa Elena, por lo que es considerada una provincia rica en bagaje cultural ancestral (Gobierno Autónomo Descentralizado Municipal de Santa Elena, 2011).

La provincia de Santa Elena ha mantenido hasta la actualidad una estructura políticaadministrativa conformada mayormente por "Comunas", donde prevalece el bienestar común, y se reconocen a sí mismos como descendientes directos de los habitantes ancestrales de esas tierras. Sin embargo, desde su fundación en 2007, la provincia de Santa Elena no ha culminado su proceso de delimitación territorial, pese a los esfuerzos del gobierno central de implementar políticas nacionales de delimitación de territorio desde el 2008 (Asamblea Nacional del Ecuador, 2010), lo que ha ocasionado conflictos territoriales y culturales dentro de esta naciente provincia, impactando negativamente su desarrollo económico y social.

Es importante mencionar, que Santa Elena es una de las provincias de mayor extensión en el país con una extensión de $3.668,90 \mathrm{~km} 2$ y una población de 144.076 habitantes lo que genera una densidad poblacional de 39 habitantes $/ \mathrm{km}^{2}$ (Gobierno Autónomo Descentralizado Municipal de Santa Elena, 2011); sin embargo, producto de la tala indiscriminada de árboles durante el siglo XIX, su suelo inició un proceso de desertificación generando escasez de tierras fértiles y menos posibilidades productivas, considerando que el Ecuador es un país agrícola (Asamblea Nacional del Ecuador, 2010). Esta escasez de tierras fértiles también ha generado conflictos sociales, pues al no existir una delimitación apropiada, las diferentes comunidades del sector, no han logrado encontrar ideales comunes para el mejoramiento de su situación social y solidaria.

El gobierno central, ha implementado varios programas para reactivar la producción agrícola en este sector del país y disolver los conflictos sociales generados de esta problemática, del cual se considera como referencia al "Proyecto Integral para el Desarrollo Agrícola, Ambiental y Social de Forma Sostenible del Ecuador" (PIDAASSE) (MAGAP, 2015). a causa de la problemática existente identificada como falta de integración de las comunidades desde sus núcleos, se considera importante abordar el tema aplicación de una "Estrategia para el mejoramiento de la Asociatividad en el Marco del Desarrollo Comunitario en las comunas de la provincia de Santa Elena, Ecuador", pues el impacto económico que se produce en la aplicación de estrategias conjuntas para el desarrollo comunitario, es favorable y brinda como resultado, una amplia gama de posibilidades favorables para los sectores deprimidos, crecimiento económico general, desarrollo industrial, etc. Donde se debe considerar que la asociatividad en sí, juega cada vez más un rol importante y activo dentro de la sociedad (Valdez, 2006); así también hay que considerar que la asociatividad se encuentra inmersa con los proyectos de desarrollo económico que puedan darse en un sector específico (Alburquerque \& otros., 2002), esto porque el espacio en donde la asociatividad facilita los mejores resultados, es el territorio visto por encima de la óptica geográfica (Areque, 2013), pues no es únicamente una superficie física, sino que es el lugar en donde se tejen las redes de relaciones; sin embargo, para su 
consolidación necesariamente tienen que girar alrededor de una variable fundamental: el "territorio geográfico" en donde se desarrolla el agrupamiento social objeto del estudio (Narváez, Fernandez, \& Senior, 2008, p. 76).

Dentro de este estudio, se analiza además, los factores involucrados en los procesos de Asociatividad, donde se ven inmersos factores económicos, culturales y sociales (Perego, 2003). A la hora de lograr un fortalecimiento efectivo del sector empresarial a través de prácticas asociativas, se debe trabajar en el accionar "equilibrado" tanto de factores económicos como no económicos (Areque, 2013).

Parte fundamental del desarrollo de grandes actividades colectivas en general, corresponde a la facilidad de todos los grupos que intervienen para generar empatía y poder asociarse eficientemente en pro de la obtención de resultados favorables para la culminación de dicha actividad, sin embargo por distintos factores económicos y no económicos existen circunstancias situacionales ajenas al proceso en sí que dificultan la obtención de dichos resultados favorables (Narváez, Fernandez, \& Senior, 2008), tal es el caso de la comuna de San Rafael en la provincia de Santa Elena, debido que por diversos problemas de varias índoles, económicos y no económicos han impedido que se logre fomentar una Asociatividad comunitaria que favorezca socialmente a esta comuna, por este motivo se plantea el problema: en la comuna de San Rafael en la provincia de Santa Elena, en el marco de aplicación del proyecto PIDAASSE, por la falta de estimulación adecuada de diversos factores económicos y no económicos no se ha logrado fomentar una "Asociatividad Comunitaria" que favorezca integralmente el desarrollo social y económico de esta comuna.

Aun cuando la problemática relacionada a la Asociatividad puede abarcar algunos escenarios (Alburquerque \& otros., 2002), la presente investigación se encuentra delimitada dentro del marco Comunitario social, específicamente en los acontecimientos que afectan al desarrollo del capital social, a la Asociatividad comunitaria, y al progreso de la comuna de San Rafael en la provincia de Santa Elena en la actualidad.

Es importante mencionar que esta realidad establecida para esta comuna, se analiza durante el proceso de implementación de un proyecto gubernamental para la activación de este sector deprimido, a través del tratamiento del suelo para que este sea apto para la agricultura de un producto importante como es el trigo (MAGAP, 2015). Se analiza entonces el impacto dentro de la comunidad con la aplicación de medidas gubernamentales y sus factores económicos, y de los demás factores no económicos implicados. Esta investigación tuvo como obejtivos (a) Identificar los factores que intervienen en los procesos de asociatividad comunitaria; (b) analizar las condiciones estimulantes para mejorar los procesos asocitativos. Y (c) determinar los factores estimulantes para el desarrollo económico de la comuna San Rafael, Provincia de Santa Elena.

\section{Revisión de literatura}

Las variables que se han determinado hacen referencia principalmente los factores que intervienen de manera directa sobre el problema de asociatividad comunitaria (Areque, 2013), existente dentro de la comuna de San Rafael en Santa Elena, estas variables son de dos tipos: (a) factores económicos y (b) factores no económicos .En la siguiente tabla se realiza la descripción 
correspondiente al tratamiento de las variables identificadas que se consideran como interventores en los procesos asociativos comunitarios:

\section{Tabla 1: Categorización De Variables}

\begin{tabular}{|c|c|c|c|c|}
\hline $\begin{array}{l}\text { Nombre de la } \\
\text { variable }\end{array}$ & Dimensiones & Ítems o indicadores & Instrumento & Unidad de Medida \\
\hline \multirow[t]{2}{*}{$\begin{array}{c}\text { Factores } \\
\text { Económicos }\end{array}$} & Competitividad & $\begin{array}{l}\text { Educación. } \\
\text { Experiencia profesional o } \\
\text { artesanal } \\
\text { Capacitación. }\end{array}$ & Entrevista & $\begin{array}{l}\text { Categorización de } \\
\text { respuestas }\end{array}$ \\
\hline & $\begin{array}{l}\text { Desarrollo } \\
\text { Económico } \\
\text { Individual } \\
\text { Desarrollo } \\
\text { Económico Colectivo }\end{array}$ & $\begin{array}{l}\text { Trabajo. } \\
\text { Crecimiento económico. } \\
\text { Crecimiento económico de } \\
\text { la comunidad. } \\
\text { Creación de Nuevas } \\
\text { Infraestructuras comunitarias. } \\
\text { Mejoramiento de } \\
\text { infraestructuras del sector. } \\
\text { Compra de nuevos activos y } \\
\text { bienes inmuebles en la } \\
\text { comunidad. }\end{array}$ & Entrevista & $\begin{array}{l}\text { Categorización de } \\
\text { respuestas } \\
\text { Categorización de } \\
\text { respuestas }\end{array}$ \\
\hline \multirow[t]{2}{*}{$\begin{array}{l}\text { Factores No } \\
\text { Económicos }\end{array}$} & Capital Social & $\begin{array}{l}\text { Estructura de la sociedad } \\
\text { Relaciones Interpersonales } \\
\text { Desarrollo Participativo } \\
\text { Trabajo en Equipo }\end{array}$ & Entrevista & $\begin{array}{l}\text { Categorización de } \\
\text { respuestas }\end{array}$ \\
\hline & Cultural & $\begin{array}{l}\text { Identidad Cultural } \\
\text { Ideología grupal } \\
\text { Confianza } \\
\text { Gobernabilidad. }\end{array}$ & Entrevista & $\begin{array}{l}\text { Categorización de } \\
\text { respuestas }\end{array}$ \\
\hline
\end{tabular}

\section{Asociatividad comunitaria}

Es importante mencionar que la Real Academia de la Lengua Española (RAE) en su 23 ava edición, emitida en octubre del 2014, no ha incluido la palabra "asociatividad" como tal dentro de sus definiciones. Este más bien constituye un concepto que se ha venido posicionando en los ámbitos investigativos, empresariales y académicos y que en los últimos años ha ganado más auge, relacionado especialmente con la búsqueda de diferentes alternativas enfocadas al mejoramiento y desarrollo empresarial de diversas naciones. Y es por este motivo que no resulta extraña esa tendencia actual de reconocer a la "Asociatividad" como una estrategia que se incorpora como requisito clave para que las empresas, no solo sobrevivan, si no que tengan la posibilidad de desarrollar ventajas competitivas, diferenciadoras y sostenibles en el tiempo (Narváez, Fernandez, \& Senior, 2008). Tal como muestra la literatura, varios estudiosos e instituciones relacionadas con la Asociatividad en general, entienden a esta de la siguiente manera: 
- Asociatividad “es un proceso orientado a la búsqueda de la cooperación interpersonal con el propósito de mejorar la gestión y la productividad en la época de la globalización" (Ministerio de Comercio, Industrias y Turismo de Colombia, 2009)

- Otro punto de vista presenta que la asociatividad es un medio que facilita la creación de ventajas competitivas gracias al trabajo conjunto de cooperación de empresas, durante las etapas que conforman la cadena de valor de un determinado producto, con la finalidad de obtener una mayor participación en los diferentes mercados donde existan oportunidades de nuevos negocios. (Romero C. , 2002).

- Según la Gobernación de Antioquia, Colombia, (2015) "La asociatividad, proceso que conduce en forma directa a la generación de sinergias, que permiten alcanzar las condiciones mínimas de mejoramiento de la calidad de vida.".

- La asociatividad "es un mecanismo de cooperación mediante el cual las pequeñas y medianas empresas unen sus esfuerzos para enfrentar las dificultades derivadas del proceso de globalización" (Liendo \& Martínez, 2001, p. 312).

\section{Asociatividad desde el ámbito empresarial}

Un aspecto importante en el marco de Asociatividad, es la relación que existe entre este término y el ámbito empresarial. En algunas de las definiciones expuestas contemplan en este ámbito que la asociatividad tiene una alta aplicabilidad exclusivamente para el sector de la pequeña y medianas empresas (PYMES). Sin embargo, ante esta tendencia, se debe tener claro que para el funcionamiento de un proceso asociativo en un territorio, es necesario y de vital importancia la participación de las grandes empresas e inclusive de instituciones gubernamentales, de darse el caso. Es decir, que la asociatividad en sí, debe englobar a todos los sectores industriales, desde el más pequeño al más grande.

Adicionalmente se considera que la mayoría de los funcionarios de las empresas grandes, las transnacionales, reconocen la necesidad de las empresas de participar en los asuntos de las comunidades dentro del territorio donde ellos se encuentran asentados (Economyc Growth, 2015)

Cuando se habla de asociatividad entre empresas también se refiere a la asociación comercial, la que se considera como una asociación voluntaria de las empresas de negocios organizados, sobre una base geográfica o industrial para promover y desarrollar oportunidades comerciales e industriales dentro de su ámbito de actuación, para expresar públicamente las opiniones de los miembros sobre asuntos de interés común (Economyc Growth, 2015).

Desde el punto de vista expuesto por Montagna, los productores más pequeños verán siempre una mejoría en mayor grado, en lo que respecta a su competitividad, pues el impulso de prácticas asociativas les permite dar frente de mejor manera a las debilidades relacionadas con las pequeñas escalas de producción y con las dificultades para acceder a nuevas y mejores tecnologías (Montagna, 2004).

Algunos de los beneficios de los que podrían gozar las empresas que buscan asociarse, sabiendo que la asociatividad genera mayores índices de desempeño en las empresas objeto de procesos asociativos, son: mayor posibilidad de ingresar a nuevos mercados o consolidarse en los 
existentes; diversificación de la demanda; posibilidad de exportar aprovechando el cambio estacional; menores costos de producción, nuevos conocimientos. Lo expuesto, hace referencia a la característica de la asociatividad que la convierte en una posibilidad estratégica, con la que un grupo de personas o empresas pueden alcanzar un objetivo común, y se pueden permitir mantener su independencia en varios aspectos, optimizando por su puesto el uso de los recursos con los que cuentan los miembros del grupo asociativo.

\section{Asociatividad Comunitaria}

El espacio en que la asociatividad logra tener mejores resultados es donde se desenvuelven las redes de relaciones interpersonales; razón por la cual cada vez más "es entendido como un espacio social que juega un papel activo" (Valdez, 2006). Y partiendo de esto es que varios autores han planteado que la asociatividad está vinculada con los procesos de desarrollo económico local, los mismos que para su consolidación deben de tener lugar en la variable fundamental, el territorio geográfico, en que se desenvuelve un agrupamiento social específico. Para poder llegar a obtener cambios considerables y permanentes en sus diferentes ámbitos que conforman a la sociedad, se requiere de la aplicación de prácticas sistemáticas y persistentes de interacción social, así se logra vincular el desarrollo económico local y el trabajo asociativo entre pobladores de un territorio específico.

Estos procesos asociativos que toman como punto de referencia el lugar geográfico alrededor del cual se agrupan las personas y empresas con el propósito de generar un objetivo común, son denominados como estrategias colectivas, basadas en los principios generales de la asociatividad. Este proceso asociativo se ve influenciado de forma directa en la actitud de las personas por trabajar de forma colectiva (Alburquerque \& otros., 2002).

Esta actitud a la que se hace referencia, en el marco territorial, es una influencia importante donde existe un crecimiento o desarrollo del territorio de forma paralela, pues es bien sabido que el crecimiento económico, puede servir a la comunidad si se mantiene la vitalidad y el carácter distintivo de la comunidad y la calidad de vida de los residentes de la comunidad (Urban sprawl, 2015). Los beneficios que viven las comunidades que atraviesan un crecimiento territorial, urbano y/o económico incluyen algunos de los siguientes puntos (Urban sprawl, 2015), lo que fomenta y promueve la asociatividad comunitaria:

- Un aumento de las oportunidades de vivienda para todos.

- El fomento de la participación ciudadana en el proceso de toma de decisiones comunitarias.

- El desarrollo de las características comunitarias que son distintivas y únicas.

- La integración de una variedad de tipos de uso del suelo en la comunidad.

- La preservación de espacios abiertos, zonas agrícolas, estructuras históricas y sitios, y los recursos ambientales que proporcionan servicios críticos a la zona.

- Un aumento de las opciones de transporte.

- El apoyo del desarrollo urbano que incluye, más que excluye, barrios existentes.

Otra visión importante a exponer dentro de esta investigación, es la relación entre asociatividad y la capacidad de emprendimiento, pues este es un factor determinante a la hora de hablar de desarrollo y progreso colectivo. 


\section{Emprendimiento}

El término emprendimiento hace referencia a aquellas personas que organizan, administran y sobre todo asumen los riesgos de un negocio o empresa (Entrepreneur, 2015) . Esta capacidad de emprendimiento aterrizada al ámbito empresarial, de acuerdo a lo propuesto por Joseph A. Schumpeter en su libro Teoría del desenvolvimiento económico, está directa y estrechamente relacionada con el trabajo innovador y creativo que realiza todo emprendedor (Schumpeter, 1976). Se puede decir entonces que, el emprendedor es definido como aquella persona con la capacidad de llevar efectivamente a la práctica nuevas combinaciones, es decir, la forma en que los factores de la producción son combinados, con el objetivo principal de obtener un determinado bien y/o servicio, pues es la calidad de su actuación que determina si el capital crece rápida o lentamente y si este crecimiento implica la innovación y el cambio (Economic growth, 2015)

Enfocando esto en el marco social comunitario, se debe considerar que dependiendo de la actitud individual y/o asociativa de cada miembro, se puede realizar un emprendimiento individual o asociativo. Un punto clave para el desarrollo de emprendimientos asociativos es que los emprendedores en sí, presentan la tendencia a tener claro quiénes son, qué saben y quiénes saben (Sarasvathy, 2001). Este último punto, es el que facilita y motiva el desarrollo de emprendimientos asociativos.

Un ejemplo claro donde se ha desarrollado un proyecto de carácter asociativo, en el cual participan diferentes actores tanto productivos, gubernamentales, educativos, es el proyecto de "Silicon Valley", en los Estados Unidos de América. En este proyecto se puede evidenciar que la asociatividad impulsada entre distintas personas relacionadas a una actividad económica específica logra producir un crecimiento económico para las comunidades asentadas en el territorio local específico (Venkataraman, 2004), de tal manera que el emprendimiento asociativo representa un factor clave a la hora de lograr una transformación regional (Venkataraman, 2004).

Con este ejemplo toma fuerza la afirmación que, desde el enfoque del estudio de "la calidad de las relaciones humanas", se debe analizar con detenimiento el comportamiento de todas las variables que intervienen debajo de la mesa, por ejemplo el capital social, pues son las personas las que determinan el funcionamiento o fracaso del emprendimiento sea individual o asociativo, de acuerdo con los objetivos definidos como meta a alcanzar.

\section{Factores Económicos que inciden en la asociatividad comunitaria}

Tal como ha demostrado la literatura en los temas anteriormente expuestos, se puede apreciar un marco de relación entre variables de tipo económicas, donde se ha buscado siempre el mejoramiento de la competitividad y el crecimiento económico, por lo que se evalúan los principales factores económicos que intervienen alrededor de un proceso asociativo.

Para iniciar, se analiza un concepto básico que engloba en su mayoría a todos estos factores, este es el concepto de "competitividad". Ésta, ha sido definida como la capacidad de esforzarse, consciente o inconscientemente para lograr cumplir un objetivo, de aquí que se 
entiende a la competitividad como parte de los pilares del fortalecimiento económico y empresarial. (Porter, 2003)

Desde el marco económico, la competitividad, es definida como la capacidad que tiene la economía en un país, para competir con la oferta de bienes y/o servicios, tanto en el mercado interno y externo. (Instituto Nacional de Estadística, Geografía e Informática, 1995)

Michael Porter, relacionando a la competitividad directamente con la definición de productividad en una relación de "causa-efecto", señala: "El único concepto significativo de la competitividad a nivel nacional es la productividad. El objetivo principal de una nación es conseguir un alto y creciente nivel de vida para sus ciudadanos. La actitud para hacerlo depende de la productividad con la que se emplee la mano de obra y el capital" (Porter, 2003); proponiendo como desafío, que, para obtener un mayor desarrollo debe existir incremento de la productividad en los sectores industriales, y una serie de capacidades necesarias para competir en mercados globalizados con niveles de complejidad aún mayores. (Porter, 2003, p. 168).

Siguiendo en la secuencia de los factores económicos que inciden a los procesos asociativos comunitarios, se menciona a continuación al desarrollo económico como un factor fundamental. El "Desarrollo Económico" es aquel proceso mediante el cual las economías simples de bajos ingresos, llegan a transformarse en economías industriales y modernas. Por lo general este término engloba la participación cualitativa, y mejoras cuantitativas de las que se generan los cambios trascendentes a nivel económico (Economic development, 2015). Desde esta perspectiva, el desarrollo económico de los países incluye entonces todas las políticas económicas, aplicadas para reanimar sectores deprimidos y/o con actividades económicas escazas y volcar su producción a niveles de industrialización junto con la innovación y desarrollo tecnológico que esto conlleva, lo que lo convierte en un asunto de interés público para el entorno local, y para la comunidad internacional, pues el desarrollo económico brinda estabilidad a un país en varios sentidos. Algunos de los factores que generan un desarrollo económico lento, es la escasez general del territorio local, por ejemplo: escasez de insumos de producción, falta de capital social, talento humano capacitado, altas tasas de desempleo, falta de políticas de protección a productores locales pues produce exceso de importaciones, entre otros (Economic development, 2015). Sin embargo, cuando se trabaja en conjunto entre las entidades públicas y privadas para tratar de erradicar estos males sociales, se puede obtener mejorías en el crecimiento económico y llevar a una sociedad de primitiva a sofisticada.

Así también el desarrollo económico, visto en su conjunto, es un factor económico que influye a los procesos de asociatividad comunitaria, acoge a la especificación del sector que se analice, y su crecimiento y desenvolvimiento dentro de una economía productiva en busca de mejoras, es decir, que para que esta tenga efecto alguno sobre los procesos asociativos, se deberá analizar el desarrollo y los factores económicos antes mencionados pero dentro de un sector de la industria en específico, con la finalidad de poder evaluar al sector, y poder aplicar adecuadamente las estrategias asociativas.

Orientando lo que se ha resumido sobre competitividad y desarrollo económico, se puede relacionarlo con los factores económicos que están inmersos en la asociatividad estrategia de cooperación colectiva, y se afirma que se coincide con varias de las propuestas que hacen 
algunos autores sobre el efecto económico que genera un proceso asociativo. (Altenburg \& Meyer-Stamer, 1999), propusieron los siguientes efectos positivos al hablar de asociatividad, en el ámbito de la asociatividad industrial:

- Posibilidad de acceder a un grupo local de mano de obra calificada;

- Atracción generada hacia los compradores de los productos ofrecidos;

- posibilidad de intercambiar información entre las empresas, instituciones e individuos, con el propósito de promover la creatividad y la innovación del sector industrial asociado.

Así también, los efectos económicos que muchos otros autores repiten, tratan de explicar los beneficios ejecutar un proceso asociativo en un determinado sector (Liendo \& Martínez, 2001): reducción de costos, acceso a tecnología y capacitación, mejor posicionamiento de mercado, acceso a grandes mercados, incremento de la productividad, acceso a recursos materiales humanos y materiales especializados, práctica de economías de escala, disponibilidad de información, captación de recursos financieros, optimización de estándares de calidad, desarrollo de nuevos productos, ventajas competitivas y mejor negociación con clientes y proveedores

\section{Factores no económicos que inciden en la asociatividad comunitaria}

Durante la elaboración de los procesos asociativos integrales, se debe mencionar que existen factores no económicos que intervienen; por ejemplo la confianza, transparencia, autogestión, cooperación, solidaridad, respeto, democracia, relaciones de poder, institucionalización, identidad cultural, compromiso con metas comunes, trabajo en equipo, etc. (Areque, 2013). Se define "Confianza" como la dependencia sobre la seguridad del carácter, habilidades, fuerza o verdad de algo o alguien, esperanza firme que se tiene de alguien o algo (RAE, 2014), lo que representa un pilar fundamental de las relaciones interpersonales de forma general, en todos los aspectos y ámbitos de la vida en relación a la sociedad. Esta investigación incluye "cultura" la misma que en antropología, geografía y otras ciencias sociales, se ve definida como, el patrón integrado del conocimiento humano, creencia y comportamiento que depende de la capacidad de aprendizaje y el conocimiento se transmite a las generaciones venideras (Culture, 2015), esto dentro de un área geográfica contigua dentro de la cual la mayoría de las sociedades comparten muchos rasgos en común (Culture area, 2015) e influye en el desempeño de las personas.

Con la finalidad de que una sociedad, o comunidad de carácter económico se pueda convertir en una ventaja competitiva, además de, aspectos técnicos y económicos, deben considerarse factores como el capital social y las relaciones interpersonales que se dan dentro de la sociedad como parte del proceso de integración. Este capital social es inherente a la estructura de las relaciones entre dos o más personas (Coleman, 2011).

El concepto de capital social sugiere que, los ciudadanos se dedican a asuntos comunitarios y públicos, esto significa que están dispuestos a transigir sobre cuestiones de polarización, donde la superación de problemas de acción colectiva se hace más fácil y las prácticas deficientes de la burocracia del sector público son menos probables. Por lo tanto, el capital social promueve ampliamente la articulación del interés y asegura la evaluación activa y 
verificación de la capacidad de respuesta de las personas e instituciones privadas y públicas (Institutional Performance, 2015).

Es importante mencionar que el capital social está conformado por tres dimensiones: Dimensión estructural, Dimensión de Relaciones y la Dimensión Cognitiva. La Dimensión estructural se refiere al patrón de conexiones entre los miembros de la comunidad. Los aspectos importantes de esta dimensión son los vínculos entre los miembros de una red social; la estructura de la sociedad se basa en la densidad, la conectividad y la jerarquía; (Aslam, Shahzad, Syed, Raza, \& Ramish, 2013). Así también la Dimensión de las Relaciones del capital social, se genera a través de relaciones activas. Se basa en las relaciones que mantienen las personas, lo que puede afectar su comportamiento por ejemplo, el respeto y la amistad, el compromiso, etc. estas relaciones son la fuente de la satisfacción de las necesidades sociales como la sociabilidad, la aprobación y el prestigio (Nahapiet \& Ghoshal, 1998) y llevan al desarrollo de la confianza e identificación con los demás miembros de un grupo social. Es imperativo mencionar además que dentro esta dimensión, dos de las cuatro dimensiones básicas del capital social definidas son el clima de confianza en las relaciones interpersonales, y capacidad de asociatividad como base de la construcción de diversas formas de cooperación entre actores. La Dimensión cognitiva, y para ello, conocer al término "Cognición" como tal, el mismo que incluye diversos tipos de pensamientos donde participan activamente la percepción, la resolución de problemas, el aprendizaje, la toma de decisiones, la lengua uso y emocional-experiencia (Cognitive science, 2015). De aquí, que la dimensión cognitiva hace referencia a la forma de interpretar y dar significado de forma compartida a las situaciones dentro de un grupo. Esta dimensión, está integrada por el lenguaje común o la visión que apoya al entendimiento grupal, para cumplir objetivos y comportamientos en el entorno social (Aslam, Shahzad, Syed, Raza, \& Ramish, 2013). La dimensión cognitiva del social capital, no es otra cosa que resultado de interacciones frecuentes que comparten las personas que realizan las mismas prácticas, y que llevan a los individuos a aprender habilidades, y conocimientos. A este concepto se adiciona lo expuesto por Kliksberg y Sen (2007) donde la conciencia cívica está relacionada, en cómo la gente actúa frente a lo considerado de interés colectivo y los valores éticos, como dimensión decisiva predominante en una sociedad, los mismos que forman parte importante del interés público y son bases para las dimensiones del capital social (Kliksberg \& Sen, 2007, pp. 265-266).

Cuando en una sociedad existe un alto nivel de capital social, se generan condiciones propicias para permitir que las comunidades y otras organizaciones se auto-organicen. Kenneth Newton, realza la importancia de lo que representa el capital social para una sociedad, "Un buen stock de capital social es prerrequisito para un efectivo sistema político" (Newton, 2001, p. 212). Se habla de capital social, cuando se habla de "aquel conjunto de normas, instituciones y organizaciones que promueven la confianza y la cooperación entre las personas, en las comunidades y en la sociedad en su conjunto" (Durston, 1999). Putnam (1993) se refiere al capital social como "ciertas características de la organización social confianza, normas y relaciones, que pueden mejorar la eficiencia de la sociedad facilitando la coordinación de acciones" esto en su obra Making Democracy Work: Civic Traditions in Modern Italy (Political Science, 2015). Otro aspecto que menciona Putnam es que "la cooperación espontánea es facilitada por el capital social", lo mismo que representa un elemento muy importante, donde se explica la relación entre capital social y asociatividad. 
Se puede incluir elementos intangibles, relacionados íntimamente con el comportamiento humano, uno de los elementos intangibles del capital social más importantes a la hora promover y consolidar un proceso asociativo es la confianza entre actores, de ahí que es considerada como el principal facilitador de la cooperación (Putnam, 1993, pp. 170-171). La confianza es la mejor garantía sobre la cual se tejen las relaciones interpersonales de los individuos de una comunidad, por lo que se aumenta espontáneamente la predisposición a compartir los recursos sin temor perjuicio. De acuerdo con Adler y Kwon, las relaciones de capital social pueden ser de tres tipos (Adler \& Kwon, 2002, p. 18):

- Relaciones cuyo origen son los intercambios de mercado, en cómo los bienes y servicios son intercambiados con dinero o a través del trueque;

- Relaciones que se originan como producto de la relación jerárquica entre actores que tienen diferentes niveles de autoridad,

- Relaciones sociales originadas en los intercambios de favores y regalos entre familiares y amigos.

Es de ahí que se indica que la asociatividad desarrollada en aglomeraciones sociales es el terreno propicio para medir y mejorar el capital social ya existente en un determinado lugar geográfico. La asociatividad y el proceso de consolidación del capital social serán más fáciles en aquellos territorios locales existe gran cantidad de las dimensiones características del capital social anotadas interiormente. En el caso de que una o varias de estas dimensiones sean una debilidad para el sitio donde se desarrolla el proceso asociativo, todas las instituciones gubernamentales y/o del sector privado y la sociedad civil, deberán atenderlo para que el proceso se cumpla.

\section{Conclusiones}

Esta investigación fue desarrollada bajo el método cualitativo, utilizando las entrevistas en profundidad como herramientas de levantamiento de información lo que permite describir el fenómeno objeto de la misma.

La primera conclusión relevante es que, los procesos asociativos comunitarios están estrechamente vinculados a la existencia de rasgos culturales asociativos previos en el entorno del territorio local, pues de no existir una cultura asociativa que busque integrar a la participación de los miembros de grupo de forma integral en beneficio de todos los que conforman la sociedad, la asociatividad en sí no tendría ningún efecto en la comunidad por lo que es de vital importancia la aplicación de una estrategia que busque estimular personalizadamente los factores que afectan a esta comuna en el marco de aplicación del Proyecto PIDAASSE.

La segunda conclusión con este proyecto se logró identificar que uno de los principales factores a estimular dentro de la comuna de San Rafael, son los factores no económicos, por lo que se expone que es importante que se busque el desarrollo del capital social, pues la ausencia de este afecta la capacidad de asociación de su pueblo, ya que al no contar con las herramientas necesarias a nivel integral de personas, estas, no serán capaces de administrar adecuadamente los recursos financieros, y se generarán discrepancias y problemas a nivel social, atentándose contra la asociatividad comunitaria. 
La tercera conclusión obtenida es que la comuna San Rafael es un candidato clave para la aplicación de una estrategia de Asociatividad Comunataria, pues ya forman parte del proyecto PIDAASSE que ha estimulado eficientemente los factores económicos, sin embargo si se aplica el nivel de motivación correcto a los factores económicos y no económicos, estos, son capaces de generar efectividad en la Asociatividad en el marco del desarrollo comunitario, produciendo beneficios y bienestar tangible e intangible para todos los miembros de la comuna San Rafael. Lo que además promueve el desarrollo económico local a través de la competitividad que se genera.

Como última conclusión, se destaca la importancia que tiene la participación de organizaciones e instituciones externas tanto públicas como privadas al momento de aplicarse medidas de mejoramiento de asociatividad comunitaria, por lo que se recomienda a las autoridades competentes, aplicar estrategias de asociatividad basadas en los principios fundamentales de incentivo y promoción para la estimulación de los factores no económicos y no únicamente a los factores económicos.

\section{Recomendaciones para futuros estudios}

No puede existir un proceso, estrategia, modelo de asociatividad, en el marco del desarrollo comunitario, donde no sean consideradas en su conjunto, todos los factores que se encuentran inmersos en el proceso, y que afectan de manera directa al territorio objeto de su aplicación. Finalmente se debe indicar que la asociatividad como estrategia para el desarrollo comunitario, es una herramienta valedera, para fortalecer a los actores económicos del desarrollo empresarial, y social de nuestro país. Por estos motivos mencionados, existe la expectativa que este trabajo de investigación sirva como base para la elaboración de políticas públicas futuras, que aseguren el bienestar comunitario; que sirva además como base para fomentar el desarrollo de programas de asociatividad integrales, para esta y todas las comunidades pertinentes dentro de nuestro país donde deben participar no unicamente el sector público si no tambien el sector privado, especialmente aquellas empresas que compran los productos de los nuevos agriculturos dentro de la comuna de San Rafael, Provinicia de Santa Elena.

Adicionalmente se ha considerado oportuno dejar planteadas algunas ideas que pueden ser ampliadas sobre la problemática revisada en este trabajo de investigación para futuras investigaciones:

- El fortalecimiento empresarial, a través de la asociatividad en la industria y el conjunto de los factores económicos y no económicos que dependen de ello.

- El emprendimiento, y su relación con el fortalecimiento del desarrollo comunitario en la asociatividad.

\section{Bibliografía}

Adler, P., \& Kwon, S.-W. (2002). Social capital: prospects for a new concept. The Academy of Managment Review, 27, 17-40.

Alburquerque, F.; Llorens, J; del Castillo, J (2002). Estudios de casos de desarrollo económico local en América Latina. Washington: Banco Interamericano de Desarrollo, (BID). 
Altenburg, T., \& Meyer-Stamer, J. (1999). How to promote clusters: policy experiences from Latin America. World Development., 27, 1693-1713.

Álvarez-Gayou, J. (2005). Cómo hacer investigación cualitativa. Fundamentos y metodología. México: Paidós.

Areque, W. (2013). Factores determinantes de la asociatividad como alternativa para el fortalecimiento de pequeñas y medianas industrias. Quito: Universidad Andina Simon Bolivar - Sede Ecuador.

Asamblea Nacional del Ecuador. (2010). Proyecto de Ley de Límites Territoriales. Quito: Asamblea Nacional del Ecuador.

Aslam, M., Shahzad, H., Syed, K., Raza, A., \& Ramish, A. (2013). Social Capital and Knowledge Sharing as Determinants of Academic Performance. Journal of Behavioral \& Applied Management.

Biocomercio del Ecuador. (2015). Biocomercio Andino. Recuperado el 20 de 08 de 2015, de http://www.biocomercioecuador.ec/biocomercio-en-el- ecuador/biodiversidad-en-el-ecuador

Coleman, J. (2011). Fundamentos de la teoría social. Madrid: Centro de Investigaciones sociologicas.

Conway, J. M., Jako, R. A., \& Goodman, D. F. (15 de Octubre de 1995). A meta- analysis of interrater and internal consistency reliability of selection interviews. Journal of Applied Psychology, págs. 565-579.

Culture area. (2015). Recuperado de Encyclopaedia Britannica Online Academic Edition. : <http://www.mdconsult.internacional.edu.ec:2172/EBchecked/topic/146313/culture-area>.

Durston, J. (1999). Construyendo capital social comunitario. Revista de la comisión Económica para América Latina (CEPAL)(69), 103-118.

Entrepreneur.

(2015).

http://www.mdconsult.internacional.edu.ec:2172/EBchecked/topic/189010/entrepreneur

Epistemology. (2015). Recuperado de Encyclopaedia Britannica Academic Online: http://www.mdconsult.internacional.edu.ec:2172/EBchecked/topic/190219/epistemology

Fraenkel, J., \& Wallen, N. (1996). Como diseñar y evaluar investigaciones en educación. New York: McGraw- Hill.

Gobierno Autónomo Descentralizado Municipal de Santa Elena. (2011). Plan De Desarrollo Y Ordenamiento Territorial 2012-2019. Santa Elena: Fundación Santiago de Guayaquil.

Gobierno Autónomo Descentralizado Provincial de Santa Elena. (2015). Historia. Recuperado el 20 de 05 de 2015, de Santa Elena: http://www.santaelena.gob.ec/index.php/historia

Instituto Nacional de Estadística, Geografía e Informática. (1995). Indicadores de Competitividad de la Economía Mexicana. . Indicadores de Competitividad de la Economía Mexicana. $N^{\circ}$ 9(9), $1-15$. 
Kliksberg, B., \& Sen, A. (2007). Primero la gente. España: Deusto.

Liendo, M., \& Martínez, A. (2001). Asociatividad, una alternativa para el desarrollo y crecimiento de las PYME. Facultad de Ciencias Económicas y Estadísticas. Rosario: Universidad del Rosario.

MAGAP. (2015). PIDAASSE. Recuperado el 28 de 05 de 2015, de Ministerio de Agricultura, Ganadería, Acuacultura y pesca.: http://www.agricultura.gob.ec/pidaasse/

Miles, M. B., \& Huberman, A. (1994). Qualitative data analysis: An expanded sourcebook . Thousand Oaks, CA: Sage.

Ministerio de Agricultura, Ganaderia, Acuacultura y Pesca. (2015). SINAGAP - Agricualtura. Recuperado el 29 de 05 de 2015, de http://sinagap.agricultura.gob.ec/maiz-duro-ce

Ministerio de Comercio, Industrias y Turismo de Colombia. (2009). Mipymes Colombia. Recuperado el 01 de 06 de 2015, de www.mipymes.gov.co

Montagna, M. (2004). Asociatividad empresarial y acceso a mercados. Chile.: Programa de Apoyo a la Microempresa Rural de America Latina y el Caribe.

Nahapiet, J., \& Ghoshal, S. (1998). Social Capital, intellectual capital, and the organizational advantage. 23(2), 242-266.

Narváez, M., Fernandez, G., \& Senior, A. (Septiembre de 2008). El desarrollo local sobre la base de la asociatividad empresarial: una propuesta estratégica. Opción., 74-92.

Newton, K. (2001). Trust, social capital, civil society and democracy. International Political Science Review., 201-214.

Nunan, D. (1992). Research Methods in Laungage learning. Cambridge: Cambridge University Press.

Padrón, J. (2007). Tendencias Epistemológicas de la Investigación Científica en el Siglo XXI. Caracas: Universidad Simón Rodríguez.

Perego, L. (2003). Competitividad a partir de los agrupamientos industriales un modelo integrado y replicable de clusters productivos. Argentina: Universidad Nacional de la Plata.

Political Science. (2015). Recuperado de Encyclopaedia Britannica Academic Online: http://www.mdconsult.internacional.edu.ec:2172/EBchecked/topic/467721/political-science

Porter, M. (2003). Ventaja Competitiva: Creación y mantenimiento de un desempeño superior. México.: CECSA.

Putnam, R. (1993). Making Democracy work: Civic Traditions in modern Italy. New Jersey: Princeton University Press.

RAE. (2014). Diccionario de la Real Academia de Lengua Española. Recuperado el 28 de 05 de 2015, de RAE: http://lema.rae.es/drae/ 
Romero, C. (2002). La Asociatividad Empresarial entre las PYME. El Salvador: Fundapyme.

Rubin, H., \& Rubin, I. (1995). Qualitative interviewing. The art of hearing data. Thousand Oaks, CA: Sage.

Statistics. (2015. ).. Recuperado, de Encyclopaedia Britannica Online Academic Edition: <http://www.mdconsult.internacional.edu.ec:2172/EBchecked/topic/564172/statistics>.

Sarasvathy, S. (2001). Causation and effectuation: toward a theoretical shift from economic inevitability to entrepreneurial contingency. The Academy of Management Review., 26, $243-$ 263.

Schumpeter, J. (1976). Teoría del desenvolvimiento económico. Mexico: Editorial Fondo de Cultura Económica.

Subsecretaría de la Pequeña y Mediana Empresa y Desarrollo Regional. (2015). Asociatividad para la exportación: claves para una estrategia exitosa. Buenos Aires: SEPYME.

Trade association. (2015). Recuperado de Encyclopaedia Britannica Online Academic Edition. : <http://www.mdconsult.internacional.edu.ec:2172/EBchecked/topic/601677/tradeassociation>.

Thesaurus. (2015). Recuperado de Encyclopaedia Britannica Academic Online Edition: http://www.mdconsult.internacional.edu.ec:2172/bps/thesaurus?query=associate

Urban sprawl. (2015). Recuperado de Encyclopaedia Britannica Online Academic Edition.: http://www.mdconsult.internacional.edu.ec:2172/EBchecked/topic/962023/urbansprawl/284710/Smart-growth-communities?anchor=ref1076177

Valdez, R. (2006). Comunidades productivas: asociatividad y producción en el territorio. Instituto para el Desarrollo y Producción Organizada, 92.

Venkataraman, S. (2004). Regional transformation through technological entrepreneurship. Journal of Business Venturing.(19), 153-167.

Vera Vélez, L. D. (2008). La Investigacion Cualitativa. Ponce: Universidad Interamericana De Puerto Rico.

Word It Out. (10 de 06 de 2015). Make a Cloud. Recuperado el 10 de 06 de 2015, de Word it out.: http://worditout.com/

Yépez Aldas, E., \& (Adaptación) Roldos Aguilera, C. (2006). Guía para la Elaboración del Proyecto de Trabajo de Grado: Procesos Paso a Paso (Adaptado C. Roldos A.). Quito, Pichincha, Ecuador: Universidad Central del Ecuador.

Zamudio Gomez, J. G. (2012). Epistemología y Educación,. Tlalnepantla: Red Tercer Milenio. 